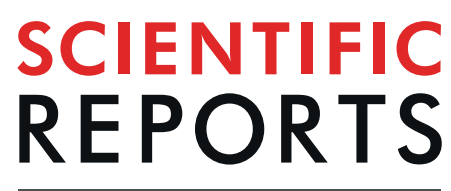

natureresearch

\title{
Radiocarbon Production Events and their Potential Relationship with the Schwabe Cycle
}

\author{
A. Scifo ${ }^{1 *}{ }^{*}$ M. Kuitems ${ }^{1}$, A. Neocleous ${ }^{2}$, B. J. S. Pope ${ }^{3}$, D. Miles ${ }^{4}$, E. Jansma ${ }^{5}$, P. Doeve ${ }^{5}$, \\ A. M. Smith ${ }^{6}$, F. Miyake ${ }^{7}{ }^{7}$ \& M. W. Dee ${ }^{1}$
}

Extreme cosmic radiation events occurred in the years $774 / 5$ and $993 / 4 \mathrm{CE}$, as revealed by anomalies in the concentration of radiocarbon in known-age tree-rings. Most hypotheses point towards intense solar storms as the cause for these events, although little direct experimental support for this claim has thus far come to light. In this study, we perform very high-precision accelerator mass spectrometry (AMS) measurements on dendrochronological tree-rings spanning the years of the events of interest, as well as the Carrington Event of $1859 \mathrm{CE}$, which is recognized as an extreme solar storm even though it did not generate an anomalous radiocarbon signature. Our data, comprising 169 new and previously published measurements, appear to delineate the modulation of radiocarbon production due to the Schwabe (11-year) solar cycle. Moreover, they suggest that all three events occurred around the maximum of the solar cycle, adding experimental support for a common solar origin.

Since the discovery of annual anomalies in the radiocarbon record, also known as Miyake Events, much effort has been undertaken to determine their cause $\mathrm{e}^{1-4}$. Detected through the analysis of tree-ring archives, the events comprise sudden and globally synchronous increases in the atmospheric concentration of radiocarbon $\left(\Delta^{14} \mathrm{C}\right)$. The first two verified anomalies occurred in the years 775 and 994 CE (henceforth Event-775 and Event-994). The uplifts were quickly attributed to increases in cosmic radiation, as radiocarbon is produced by means of nuclear reactions associated with the cosmic ray cascade ${ }^{5,6}$. The rate of production is proportional to the cosmic ray flux entering the Earth's magnetosphere, which is in turn modulated by the interplanetary magnetic field in accordance with the Schwabe cycle. In fact, this solar cycle is inversely related to radiocarbon production as, during periods of maximal solar activity, the shielding of cosmic rays by the solar plasma in the interplanetary and the geomagnetic fields is enhanced. In such cases, a decrease in radiocarbon production occurs, and vice versa ${ }^{7-9}$. This sinusoidal variation has already been measured in tree-ring samples by various groups, and exhibits a peak-to-trough amplitude of around $4-6 \% 0^{8,10-12}$. Despite this anticorrelation, the most widely accepted theory for the cause of the anomalous increases in $\Delta^{14} \mathrm{C}$ is extreme solar storms ${ }^{3,13}$. This is largely because of the occurrence frequency of the events, as several have now been attested and others predicted during the Holocene $e^{4,14,15}$, but it is also the conclusion of modelling studies of isotopic signatures in natural archives ${ }^{16}$. However, to date few direct connections have been made between the Miyake Events and established solar behavior, or instrumentally monitored solar storms. Indeed, some analyses still contradict the claim of a solar origin altogether ${ }^{17,18}$. Here, our results suggest that Event-775 and Event-994 both occurred during the period of the 11-year solar cycle when the Sun was most active. This provides additional support for the hypothesis that these anomalies were instigated by powerful solar emissions.

The most intense solar storm to hit Earth in modern times was the Carrington Event of September 1859 CE. In this case, the solar flare itself was directly witnessed ${ }^{19}$, and the magnetic excursions recorded at Kew Observatory in London outstripped measurement capacity ${ }^{20}$. The so-called magnetic crochet associated with the impact of

\footnotetext{
${ }^{1}$ University of Groningen, Centre for Isotope Research, Nijenborgh 6, 9747AG, Groningen, The Netherlands. ${ }^{2}$ University of Cyprus, Department of Computer Science, 1 University Avenue, 2109, Aglantzia, Cyprus. ${ }^{3}$ NASA Sagan Fellow, Center for Cosmology and Particle Physics and Center for Data Science, New York, NY, USA. ${ }^{4}$ Oxford University, Oxford Dendrochronology Laboratory, Mill Farm, Mapledurham, Oxfordshire, RG4 7TX, United Kingdom. ${ }^{5}$ Cultural Heritage Agency of The Netherlands, Smallepad 5, 3811 MG, Amersfoort, The Netherlands. ${ }^{6}$ Australian Nuclear Science and Technology Organisation (ANSTO), New Illawarra Rd, Lucas Heights, NSW, 2234, Australia. ${ }^{7}$ Nagoya University, Institute for Space-Earth Environmental Research, Chikusa-ku, Nagoya, 464-8601, Japan. *email: a.scifo@rug.nl
} 


\begin{tabular}{|c|c|c|c|c|c|}
\hline \multicolumn{3}{|c|}{ Sample A (Oak) - Carrington } & \multicolumn{3}{|c|}{ Sample B1 (Juniper) - Event-775 } \\
\hline $\begin{array}{l}\text { Calendar Age } \\
\text { (year CE) }\end{array}$ & \begin{tabular}{|l}
${ }^{14} \mathrm{C}$ age \\
(yr BP)
\end{tabular} & $\begin{array}{l}\Delta^{14} \mathrm{C} \\
(\%)\end{array}$ & $\begin{array}{l}\text { Calendar Age } \\
\text { (year CE) }\end{array}$ & \begin{tabular}{|l}
${ }^{14} \mathrm{C}$ age \\
$(\mathrm{yr} B \mathrm{BP})$
\end{tabular} & $\begin{array}{l}\Delta^{14} \mathrm{C} \\
(\%)\end{array}$ \\
\hline $1853^{*}$ & $126 \pm 11$ & $-3.94 \pm 1.40$ & 756 & $1350 \pm 15$ & $-23.35 \pm 1.82$ \\
\hline 1855 & $137 \pm 16$ & $-5.55 \pm 2.01$ & 758 & $1344 \pm 15$ & $-22.86 \pm 1.82$ \\
\hline $1856^{*}$ & $149 \pm 11$ & $-7.03 \pm 1.36$ & 759 & $1360 \pm 25$ & $-24.92 \pm 3.03$ \\
\hline 1857 & $119 \pm 13$ & $-3.56 \pm 1.55$ & $762-763$ & $1305 \pm 16$ & $-18.63 \pm 1.98$ \\
\hline 1858 & $111 \pm 13$ & $-2.69 \pm 1.55$ & 765 & $1316 \pm 16$ & $-20.27 \pm 1.98$ \\
\hline $1859 *$ & $144 \pm 8$ & $-6.89 \pm 0.99$ & 766 & $1311 \pm 15$ & $-19.78 \pm 1.83$ \\
\hline $1860 *$ & $130 \pm 10$ & $-5.03 \pm 1.24$ & 768 & $1324 \pm 16$ & $-21.61 \pm 1.97$ \\
\hline 1861 & $145 \pm 13$ & $-7.26 \pm 1.54$ & 769 & $1308 \pm 15$ & $-19.77 \pm 1.83$ \\
\hline $1862^{*}$ & $133 \pm 9$ & $-5.89 \pm 1.08$ & 771 & $1326 \pm 24$ & $-22.20 \pm 2.89$ \\
\hline 1863 & $158 \pm 13$ & $-9.10 \pm 1.54$ & 772 & $1320 \pm 18$ & $-21.59 \pm 2.13$ \\
\hline $1864^{*}$ & $140 \pm 11$ & $-7.00 \pm 1.40$ & 775 & $1172 \pm 16$ & $-3.76 \pm 2.01$ \\
\hline $1865^{*}$ & $137 \pm 9$ & $-6.75 \pm 1.08$ & $777^{*}$ & $1177 \pm 11$ & $-4.62 \pm 1.39$ \\
\hline 1866 & $119 \pm 13$ & $-4.64 \pm 1.54$ & & & \\
\hline \multicolumn{3}{|c|}{ Sample B2 (Oak) - Event-775 } & \multicolumn{3}{|c|}{ Sample C (Juniper) - Event-994 } \\
\hline $\begin{array}{l}\text { Calendar Age } \\
\text { (year CE) }\end{array}$ & $\begin{array}{l}{ }^{14} \mathrm{C} \text { age } \\
(\mathrm{yr} \mathrm{BP})\end{array}$ & $\begin{array}{l}\Delta_{(\%)}^{14} \mathrm{C} \\
(\%)\end{array}$ & $\begin{array}{l}\text { Calendar Age } \\
\text { (year CE) }\end{array}$ & \begin{tabular}{|l}
${ }^{14} \mathrm{C}$ age \\
(yr BP)
\end{tabular} & $\begin{array}{l}\Delta^{14} \mathrm{C} \\
(\%)\end{array}$ \\
\hline 770* & $1290 \pm 7$ & $-17.72 \pm 0.86$ & 976 & $1136 \pm 15$ & $-23.32 \pm 1.82$ \\
\hline $771^{*}$ & $1278 \pm 12$ & $-16.32 \pm 1.45$ & 978 & $1108 \pm 15$ & $-20.15 \pm 1.83$ \\
\hline $772^{*}$ & $1281 \pm 6$ & $-16.87 \pm 0.76$ & 980 & $1113 \pm 18$ & $-20.99 \pm 2.13$ \\
\hline $773^{*}$ & $1308 \pm 11$ & $-20.20 \pm 1.39$ & 982 & $1143 \pm 16$ & $-24.88 \pm 1.97$ \\
\hline 774 & $1285 \pm 25$ & $-17.56 \pm 3.06$ & 984 & $1120 \pm 16$ & $-22.32 \pm 1.98$ \\
\hline $775^{*}$ & $1211 \pm 15$ & $-8.63 \pm 1.85$ & $986^{*}$ & $1102 \pm 11$ & $-20.36 \pm 1.37$ \\
\hline $776^{*}$ & $1142 \pm 15$ & $-0.19 \pm 1.87$ & 988 & $1116 \pm 18$ & $-22.31 \pm 2.13$ \\
\hline $777^{*}$ & $1153 \pm 14$ & $-1.65 \pm 1.69$ & 989-991* & $1096 \pm 12$ & $-20.11 \pm 1.52$ \\
\hline $778^{*}$ & $1168 \pm 10$ & $-3.66 \pm 1.25$ & 993 & $1047 \pm 16$ & $-14.47 \pm 1.99$ \\
\hline \multirow[t]{2}{*}{ 779* } & $1176 \pm 14$ & $-4.78 \pm 1.69$ & 995 & $1035 \pm 16$ & $-13.23 \pm 1.99$ \\
\hline & & & 997 & $1045 \pm 16$ & $-14.70 \pm 1.99$ \\
\hline
\end{tabular}

Table 1. Results of the radiocarbon analysis over the Carrington Event, Event-775 and Event-994. *Years where pretreatments were repeated and duplicate measurements obtained and averaged (See S1, S2 and S3, SI).

the initial soft x-ray/extreme ultra-violet flux on the ionosphere remains one of the largest ever reported for the mid-latitudes; and intensified aurorae, instigated by the geomagnetic disturbance, were observed as far south as the tropics ${ }^{20}$. Further consequences, such as malfunctions to telegraph systems, were also recorded world-wide ${ }^{21}$. Despite these dramatic impacts, the Carrington Event left no detectable imprint on the atmospheric radiocarbon record $^{22}$. However, continuous and reliable observations of the solar cycle were already being made at the time of the Carrington Event. This was achieved by recording the number of sunspots (known as the International Sunspot Number, ISN). Hence, it was quickly established that the storm took place six months prior to the maximum activity of the solar cycle.

Historical solar activity may be elucidated by carrying out very high precision AMS measurements on series of annual tree-rings spanning the events in question. In our study, Event-775 and Event-994 are analyzed at levels of precision that allow us to claim evidence of the modulation of atmospheric radiocarbon due to the 11-year Schwabe cycle ${ }^{11,23,24}$. As a result, we are able to assign a possible timing for these events in relation to the solar cycle, an outcome that has several important implications. Firstly, if each event occurred around the maximum of the solar cycle, like the Carrington Event, this would provide additional support for a common solar origin. To elaborate, solar flares and coronal mass ejections (CMEs) are in general more frequent and more intense during times of highest solar activity. For example, the recorded ISN and the number of higher energy flares (M-class and $\mathrm{X}$-class flares) are very well correlated $\left(\mathrm{r}=0.95, \mathrm{r}^{2}=0.90\right)$; although, significant flares can occur throughout the whole period $^{25}$. Secondly, establishing whether the Carrington Event, Event-775 and Event-994 all occurred at the same point on the Schwabe cycle may suggest further shared characteristics, such as whether the mechanisms that drove Event-775 and Event-994 can be regarded as extreme versions of those that initiated Carrington.

\section{Results}

In this study, we make use of 169 radiocarbon data; of these, 76 are new measurements and 93 come from previously published datasets. The suite of new results, averaged per year, is given in Table 1 . The Supplementary Information (SI) contains the full set of newly obtained data (Table S1), the previously published datasets (Table S2) and details about how duplicate measurements were dealt with, including the outputs of chi-squared statistical comparisons (Table S3). The new results were obtained at an average precision of $1.71 \%$ o $(1 \sigma)$ per year, with reported uncertainties encompassing counting statistics, normalization and sample preparation calculated in accordance with standard data reduction procedures ${ }^{26}$. 

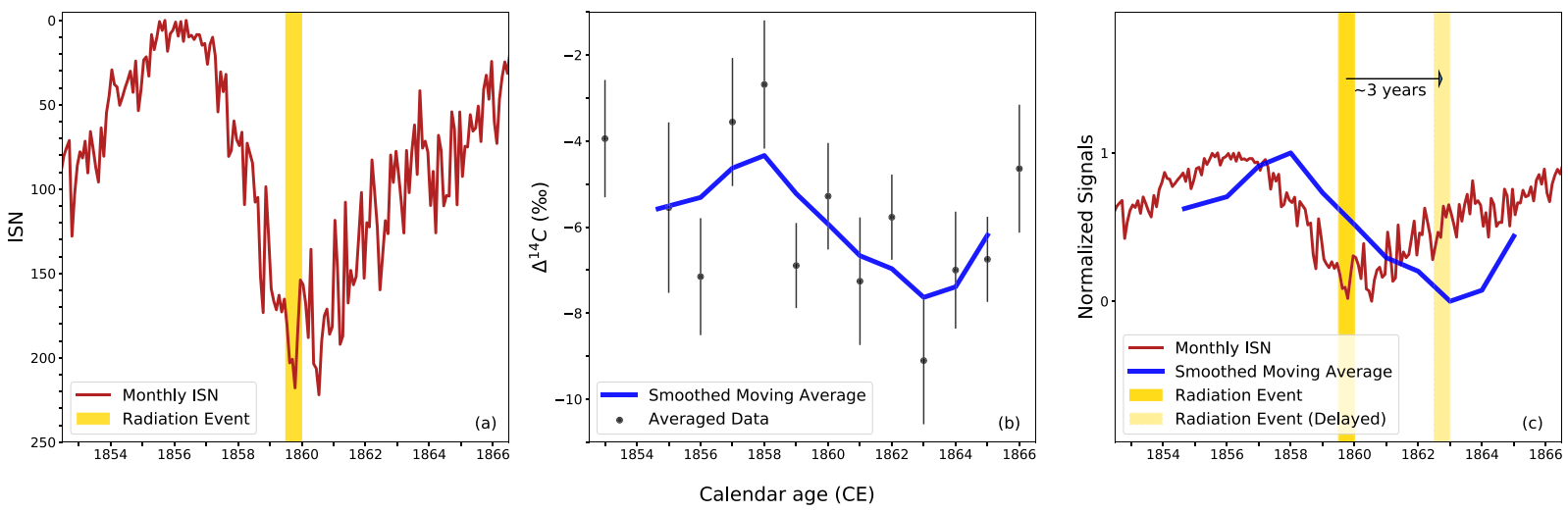

Figure 1. Left, monthly ISN (red, see S5 (SI) for further details) and timing of the radiation event (yellow). Center, averaged results of the 21 measurements on Sample A (1-sigma error bars). Smoothed moving average (blue) obtained using a 3-year moving average smoothed by a Savitzky-Golay filter ${ }^{44}$. Right, normalized monthly ISN (red) and smoothed moving average (blue). The delay between the two signals ( $\sim 3$ years) is an approximation of the radiocarbon atmospheric residence time.

Investigation of radiocarbon modulation due to the solar cycle has previously been conducted in different ways. Burchuladze et al..$^{27}$ directly compared the ISN with radiocarbon measurements on vintage wine samples ${ }^{27}$; Stuiver \& Braziunas ${ }^{10}$ applied a cubic spline interpolation to annual tree-ring data and evaluated the residuals between the subsequent fit and a moving average ${ }^{10}$; and Gütler et al. ${ }^{11}$ used a band-pass filter to analyze the signal in order to extract the hidden periodicities from tree-ring data ${ }^{11}$. Of these three approaches, the first one can only be applied over the time since the ISN has been recorded, therefore we make use of it only in the case of the Carrington Event. Of the other two, we concentrate on the band-pass filter, but we have also completed some analyzes using the residuals from the spline interpolation, which can be found in S7 (SI). In relation to the filter, we used a Butterworth band-pass filter ${ }^{28}$ which was designed to extract periodicities between 8 and 20 years (see Güttler et al. ${ }^{11}$ ). It is important to note that applying a digital filter to a dataset will undesirably but unavoidably introduce an offset, as the output of the filter will be altered from the input signal ${ }^{29}$. This happens because using a filter with fixed cut-off frequencies and order will shift sinusoids of different frequencies by different amounts. In our case, the filter will introduce a variable offset of 3 years or less.

Comparing $\Delta^{14} \mathrm{C}$ with ISN over the Carrington Event. For this study, we made use of 21 radiocarbon measurements over 13 single-year tree-rings. Multiple measurements were performed on 7 of the samples, and the results were averaged (see Table S1a, SI). Due to the high precision of the radiocarbon measurements obtained, a broad modulation of the $\Delta^{14} \mathrm{C}$ data can be observed, with a peak-to-trough amplitude of about $5 \%$. This value is consistent with the effect of solar modulation pointed out in previous analyzes of the Schwabe cycle ${ }^{8,11,30-32}$. Moreover, when presented together with the ISN data (see Fig. 1), it is clear that the ${ }^{14} \mathrm{C}$ data follow the same pattern (the ISN axis is inverted to account for the inverse relationship between the two parameters). As also expected, the two signals were not perfectly aligned, as the sunspot record reflects immediate solar behavior, while the radiocarbon record is delayed due to the so-called residence time of the isotope in the atmosphere. By measuring the cross-correlation between the normalized $\Delta^{14} \mathrm{C}$ and the normalized ISN data, one can estimate the delay time between the two signals. Here this residence time is estimated to be $3 \pm 1$ years, which is in good agreement with previous estimates ${ }^{33,34}$. As can be seen, our $\Delta^{14} \mathrm{C}$ data approximately follow the sinusoidal profile of the solar cycle, and hence mimic the variability of incoming solar radiation.

Event-775. For this study, we made use of a total of 58 radiocarbon measurements over 32 single-year tree-rings. Of these, 15 are new measurements from sample B1, 27 are from sample B2, and 16 were previously produced by Miyake et al. ${ }^{2}$. Multiple measurements were performed on 11 of the 32 single-year tree-rings, and the results were averaged for each year (see Tables S1b and S1c, SI).

The radiocarbon results are in close agreement with previous measurements over the event ${ }^{1,3}$. The outcome of our analysis of the data is shown in Fig. 2. From the averaged $\Delta^{14} \mathrm{C}$ data leading up to the spike in the year 775 $\mathrm{CE}$, a sinusoidal pattern is clearly evident. This is especially true for the decades immediately preceding the event, where we achieve the highest precisions. We observe approximately four cycles within this interval of 45 years, which have a peak-to-trough amplitude that is similar to the Carrington Event $(\sim 5 \%)$, and a period of approximately 11 years in length. This pattern was accentuated by the residuals from the cubic interpolation (see S7, SI). Thus, we note that Event-775, like the Carrington Event, appears to occur when solar activity is at its maximum (Fig. 2).

Event-994. For this study, we made use of a total of 90 radiocarbon measurements over 48 single-year tree-rings, although on this occasion, due to availability of tree rings, the radiation event is located towards the middle of the series. Of these, 13 are new measurements from sample C, including 2 replicated measurements (see Table S1d, SI). This data was complemented by 13 results from Damon et al. ${ }^{35} ; 7$ from Dee et al. ${ }^{36} ; 25$ from Menjo et al. ${ }^{37}$; and 32 from Miyake et al. ${ }^{23,35-37}$. 

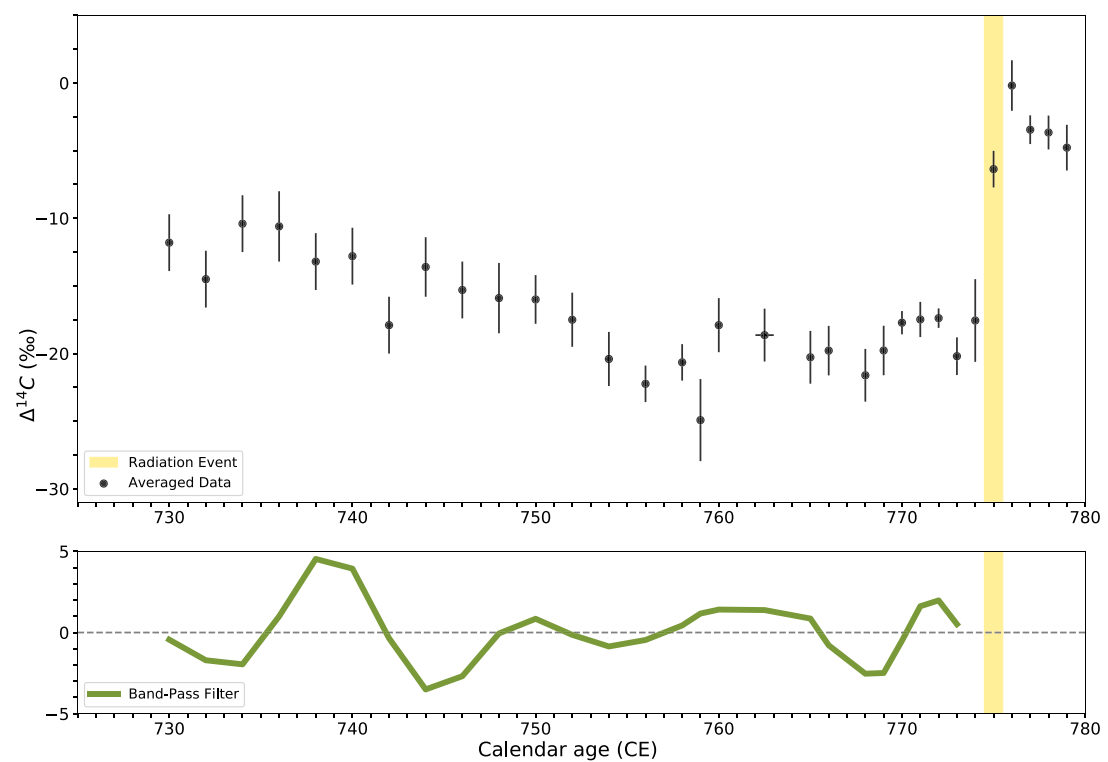

Figure 2. Results of the analysis over the Event-775. In the top row, averaged data points with 1-sigma error bars (black) and timing of the radiation event (yellow). In the bottom row, the result of a Butterworth band-pass filter applied to the $\Delta^{14} \mathrm{C}$ data (green). The band-pass filter is only applied to the data prior to the spike, in order to not affect the periodicity.
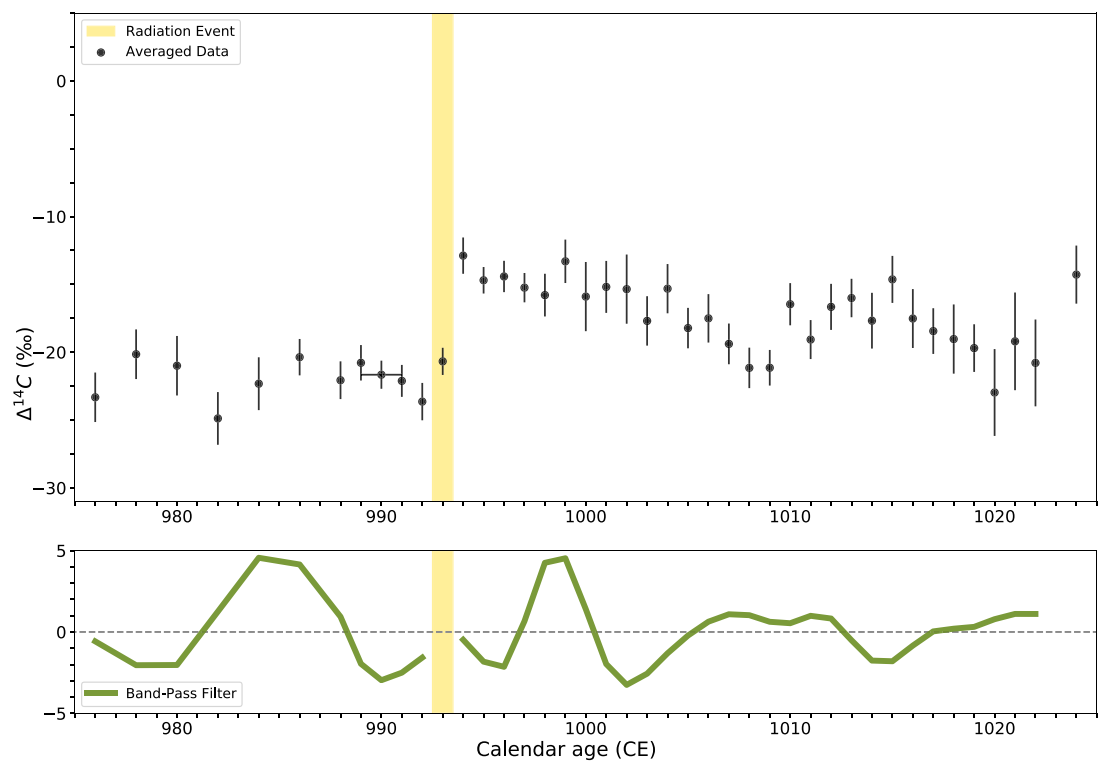

Figure 3. Results of the analysis over the Event-994. In the top row, averaged data points with 1-sigma error bars (black) and timing of the radiation event (yellow). In the bottom row, the result of a Butterworth band-pass filter applied to the $\Delta^{14} \mathrm{C}$ data (green). The band-pass filter is applied separately before and after the spike.

Once more, the radiocarbon results of the newly measured data are in good agreement with previously published results ${ }^{2}$. Individual tree-rings prior to the Event-994 spike were sampled every other year, apart from the last data point (989-991 CE), which came from three tree-rings too narrow to separate. Our band-pass filter analysis of the data is shown in Fig. 3, while the spline residuals are presented in S7 (SI). From the averaged $\Delta^{14} \mathrm{C}$ data leading up to and after the spike, a sinusoidal pattern is again evident. They indicate that approximately 4.5 cycles occurred within this interval of 50 years. It should be noted that the band-pass filter was applied separately before and after the spike, in order to limit the disruption caused by the sudden increase in $\Delta^{14} \mathrm{C}$. However, for at least a decade afterwards the output is still clearly influenced by the radiocarbon spike (Fig. 3 ).

It is also important to point out that this peak occurs in $993 \mathrm{CE}$, one year before the recognized date for Event994, but this is consistent with several other studies (see Büntgen et al. ${ }^{38}$ ). In this case, we also tentatively interpret 


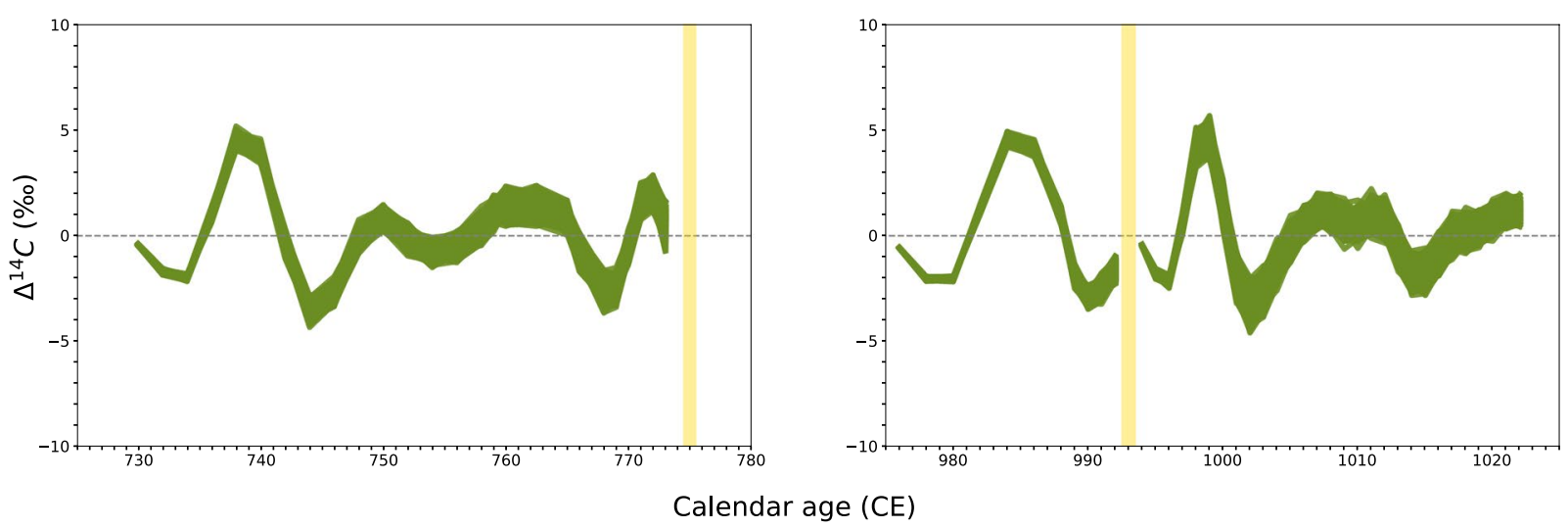

Figure 4. Butterworth band-pass filter applied to Monte Carlo resampling |(1,000 times) of the normally distributed experimental data analyzed in Figs. 2 and 3. The yellow vertical band represents the year in which the event occurred, which was at least one year before the peak in $\Delta^{14} \mathrm{C}$, for Event-775 and Event-994. For both events, the intersection between the yellow vertical band and the data interpolation corresponds to maximum activity of the sun within the Schwabe cycle or lowest radiocarbon production rate.

the patterns in our data as evidence of the solar modulation of atmospheric radiocarbon production. Therefore, we note that Event-994 also appears to occur when solar activity is at its maximum (Fig. 3$)^{38}$.

Monte Carlo resampling. In order to establish if the results of our numerical analyzes were influenced by a possible low signal-to-noise ratio in our datasets, we performed Monte Carlo simulations in which we randomly resample our datasets 1,000 times, assuming the data was Normally distributed. Then, we applied the same Butterworth band-pass filter over the resampled datasets. In Fig. 4, we report the outcome of these analyzes for both the events in terms of $\Delta^{14} \mathrm{C}$ versus sample growth year (CE). For Event-775 and Event-994, the results are in accordance with the outputs shown in Figs. 2 and 3, albeit more distinct and robust. Hence, we conclude that the Carrington Event, Event-775 and Event-994 all appear to have occurred near the point of maximum activity of the solar cycle.

\section{Discussion}

Our results present a coherent picture across all three solar events. We observed a moderate variation in radiocarbon over the Carrington Event of $1859 \mathrm{CE}$; our new and previously published data over Event-775 followed a sinusoidal pattern with peak-to-trough amplitude of about 5\%, and a periodicity of about 12 years; likewise, our Event-994 data varied with a 5\% amplitude and period of around 11 years. Although it cannot be stated categorically, we believe that the most parsimonious explanation for the undulating pattern in all our datasets is the solar modulation of radiocarbon production. No other repeating process of this same magnitude and duration is easily conceivable. This finding is best exemplified by the Monte Carlo resampling, as it accounts for the inherent variability in estimates of radiocarbon concentration.

In all three cases, the radiation events occur in close proximity to the point of maximum activity of the 11-year solar cycle. Although it did not leave any radiocarbon signature, the Carrington Event of 1859 CE was already known to have occurred around the point of maximum activity of the solar cycle, due to contemporary accounts of the sunspot record. Direct comparison between our new $\Delta^{14} \mathrm{C}$ data over the Carrington Event and such sunspot counts also allowed us to make an estimation of $3 \pm 1$ years for the residence time of radiocarbon in the atmosphere at this time. In relation to Event-775 and Event-994, our data also show that the radiation events occurred when the sun was at its most active, and radiocarbon production exhibited a local minimum.

In summary, our finding strengthens the likelihood of a solar origin for Event-775 and Event-994, and provides valuable experimental evidence of a link between them and the Carrington Event.

\section{Methods}

Four different dendrochronologically dated wood samples were obtained and pretreated, each containing distinct annual growth rings spanning the years of the three different events. Sample A, covering the Carrington Event, was a piece of oak from southern England (see Fig. 5). Samples B1 and C, spanning Event-775 and Event-994, respectively, were two pieces of juniper, which grew in the Sierra Nevada Mountains, California, at an altitude of about $3000 \mathrm{~m}$. These samples were obtained from the Oxford Dendrochronology Laboratory, England. A further oak sample which also traversed Event-775, B2, was obtained from the Cultural Heritage Agency of The Netherlands. The rings corresponding to the years of interest in sample B1 were quite thin, while the ones from Sample C were somewhat irregular in shape, which made separation challenging. Nonetheless, the possibility of material from adjacent rings being mixed together was considered minimal.

The photosynthetic uptake of ${ }^{14} \mathrm{CO}_{2}$ is indistinguishable from its stable isotopic analogues, save a degree of readily correctable mass-dependent fractionation. Much of the carbon absorbed is immediately locked into the cellulosic structure of the growth-rings, and chemical exchange between rings is negligible ${ }^{39,40}$. Hence, the optimal extract for reconstructing past $\Delta^{14} \mathrm{C}$ levels is the fraction known as alpha-cellulose. The method used for such 

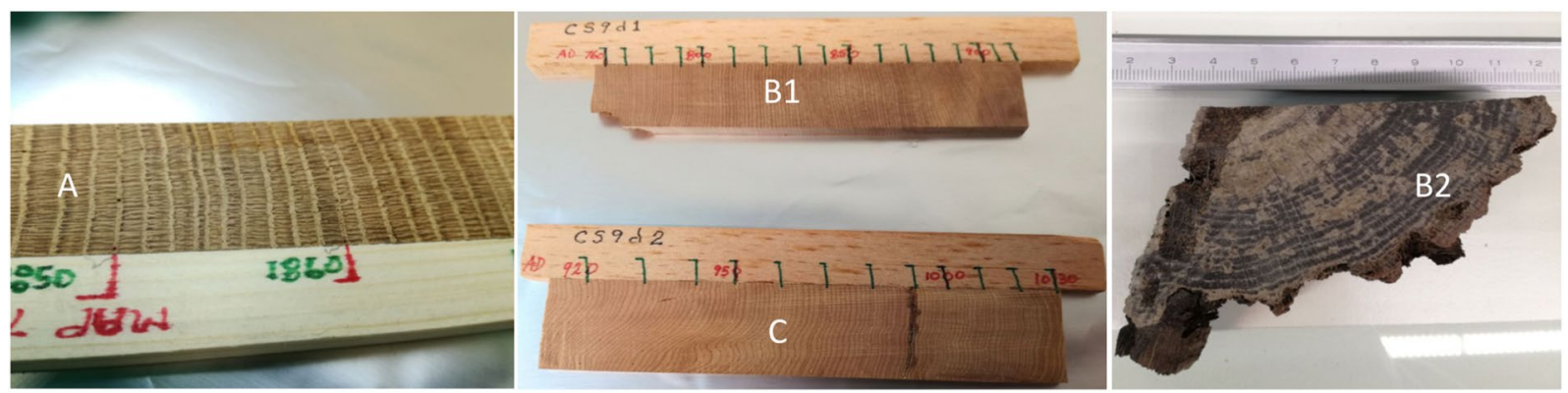

Figure 5. Left, Sample A, the oak piece analyzed for the Carrington event. As is evident in the photo, the wood exhibits marked and distinct annual growth rings. Center, Samples (B1) (above) and (C) (below), juniper wood analyzed for the study of Event-775 and Event-994. The growth rings are once more evident but finer and at times more warped than in Sample (A). Right, Sample (B2), oak wood analyzed for Event-775. Growth rings are marked and distinct over most of the sample.

extractions at Groningen is described in detail by Dee et al. ${ }^{41}$, so only a brief summary is presented here. The wood samples first undergo a physical preparation procedure, including elimination of extraneous soil and particulates from the bulk material, cutting each growth ring from the main sample, and then slicing or crushing each into small fragments. The sample is then chemically pretreated with an intense Acid-Base-Acid procedure, namely $\mathrm{HCl}\left(5.47 \% \mathrm{w} / \mathrm{vol}(1.5 \mathrm{M}), 80^{\circ} \mathrm{C}, 20 \mathrm{~min}\right) ; \mathrm{NaOH}(17.5 \% \mathrm{w} / \mathrm{vol}, 60 \mathrm{~min}$, RT) with ultra-sonication under an $\mathrm{N}_{2}$ atmosphere; $\mathrm{HCl}\left(5.47 \% \mathrm{w} / \mathrm{vol}(1.5 \mathrm{M}), 80^{\circ} \mathrm{C}, 20 \mathrm{~min}\right)$, followed by a strong acidified oxidant $\left(\mathrm{NaClO}_{2}\right.$, $1.5 \% \mathrm{w} / \mathrm{vol}$ in $\left.\mathrm{HCl}(0.06 \mathrm{M}), 80^{\circ} \mathrm{C}, 20 \mathrm{hrs}\right)$, in order to extract the alpha-cellulose fraction. Each step is separated by a thorough rinse to neutrality with deionized and decarbonized water. Next, the alpha-cellulose fraction is combusted, and the $\mathrm{CO}_{2}$ liberated cryogenically trapped, reduced to graphite and pressed into Al cathodes ${ }^{42,43}$. The radiocarbon content of graphite extracted from each sample is then determined by AMS (200kV MICADAS, Ionplus). In order to achieve very high precision measurements, the samples are measured for longer periods than usual.

\section{Data availability}

All data generated or analyzed during this study are included in this published article (and its Supplementary Information files).

Received: 19 August 2019; Accepted: 24 October 2019;

Published online: 19 November 2019

\section{References}

1. Miyake, F., Nagaya, K., Masuda, K. \& Nakamura, T. A signature of cosmic-ray increase in AD $774-775$ from tree rings in Japan. Nature 486, 240-242 (2012).

2. Miyake, F., Masuda, K. \& Nakamura, T. Another rapid event in the carbon-14 content of tree rings. Nat. Commun. 4, 1748 (2013).

3. Usoskin, I. G. et al. The AD775 cosmic event revisited: the Sun is to blame. Astron. Astrophys. 552, L3 (2013).

4. Wang, F. Y., Yu, H., Zou, Y. C., Dai, Z. G. \& Cheng, K. S. A rapid cosmic-ray increase in BC 3372-3371 from ancient buried tree rings in China. Nat. Commun. 8, 1487 (2017).

5. De Vries, H. \& Barendsen, G. W. Measurements of age by the Carbon-14 technique. Nature 174, 1138 (1954).

6. Lingenfelter, R. E. Production of carbon 14 by cosmic-ray neutrons. Rev. Geophys. 1, 35 (1963).

7. Stuiver, M. Variations in radiocarbon concentration and sunspot activity. J. Geophys. Res. 66, 273-276 (1961).

8. Damon, P. E., Long, A. \& Wallick, E. I. On the magnitude of the 11-year radiocarbon cycle. Earth Planet. Sci. Lett. 20, 300-306 (1973).

9. Damon, P. E. \& Linick, T. W. Geomagnetic-Heliomagnetic Modulation of Atmospheric Radiocarbon Production. Radiocarbon 28, 266-278 (1986).

10. Stuiver, M. \& Braziunas, T. F. Anthropogenic and solar components of hemispheric ${ }^{14}$ C. Geophys. Res. Lett. 25, 329-332 (1998).

11. Güttler, D., Wacker, L., Kromer, B., Friedrich, M. \& Synal, H.-A. Evidence of 11-year solar cycles in tree rings from 1010 to 1110 AD - Progress on high precision AMS measurements. Nucl. Instruments Methods Phys. Res. Sect. B Beam Interact. with Mater. Atoms 294, 459-463 (2013).

12. Friedrich, R. et al. Annual 14C tree-ring data around 400 AD: mid- and high-latitude records. Radiocarbon 00, 1-12 (2019).

13. Melott, A. L. \& Thomas, B. C. Causes of an AD 774-775 14C increase. Nature 491, E1-E2 (2012).

14. O'Hare, P. et al. Multiradionuclide evidence for an extreme solar proton event around 2,610 B.P. ( 660 BC). Proc. Natl. Acad. Sci. 116, 5961-5966 (2019).

15. Neocleous, A., Azzopardi, G., Kuitems, M., Scifo, A. \& Dee, M. Trainable Filters for the Identification of Anomalies in Cosmogenic Isotope Data. IEEE Access 7, 24585-24592 (2019).

16. Mekhaldi, F. et al. Multiradionuclide evidence for the solar origin of the cosmic-ray events of AD 774/5 and 993/4. Nat. Commun. 6, 8611 (2015).

17. Pavlov, A. K. et al. Gamma-ray bursts and the production of cosmogenic radionuclides in the Earth's atmosphere. Astron. Lett. 39, 571-577 (2013).

18. Neuhäuser, R. \& Hambaryan, V. V. A solar super-flare as cause for the ${ }^{14} \mathrm{C}$ variation in AD $774 / 5$ ? Astron. Nachrichten 335, $949-963$ (2014).

19. Carrington, R. C. Description of a Singular Appearance seen in the Sun on September 1, 1859. Mon. Not. R. Astron. Soc. 20, 13-15 (1859). 
20. Cliver, E. W. \& Svalgaard, L. The 1859 Solar-Terrestrial Disturbance And the Current Limits of Extreme Space Weather Activity. Sol. Phys. 224, 407-422 (2004).

21. Tsurutani, B. T., Gonzalez, W. D., Lakhina, G. S. \& Alex, S. The extreme magnetic storm of 1-2 September 1859. J. Geophys. Res. 108, 1268 (2003).

22. Stuiver, M., Reimer, P. J. \& Thomas, F. Braziunas. High-precision radiocarbon age calibration for terrestrial and marine samples. Radiocarbon 40, 1127-1151 (1998).

23. Miyake, F., Masuda, K. \& Nakamura, T. Lengths of Schwabe cycles in the seventh and eighth centuries indicated by precise measurement of carbon-14 content in tree rings. J. Geophys. Res. Sp. Phys. 118, 7483-7487 (2013).

24. Eastoe, C. J., Tucek, C. S. \& Touchan, R. $\triangle 14 \mathrm{C}$ and $813 \mathrm{C}$ in Annual Tree-Ring Samples from Sequoiadendron Giganteum, AD 998-1510: Solar Cycles and Climate. Radiocarbon 61, 661-680 (2019).

25. Hathaway, D. H. The Solar Cycle. Living Rev. Sol. Phys. 12, 4 (2015).

26. Mook, W. G. \& van der Plicht, J. Reporting Radiocarbon Activities and Concentrations. Radiocarbon 41, 227-239 (1999).

27. Burchuladze, A. A., Pagava, S. V., Povinec, P., Togonidze, G. I. \& Usacev, S. Radiocarbon variations with the 11-year solar cycle during the last century. Nature 287, 320-322 (1980).

28. Butterworth, S. On the Theory of Filter Amplifiers. Exp. Wirel. Wirel. Eng. 7, 536-541 (1930).

29. Manal, K. \& Rose, W. A general solution for the time delay introduced by a low-pass Butterworth digital filter: An application to musculoskeletal modeling. J. Biomech. 40, 678-681 (2007).

30. Stuiver, M. Carbon-14 Content of 18th- and 19th-Century Wood: Variations Correlated with Sunspot Activity. Science 149, 533-535 (1965).

31. Povinec, P., Burchuladze, A. A. \& Pagava, S. V. Short-Term Variations in Radiocarbon Concentration with the 11-Year Solar Cycle. Radiocarbon 25, 259-266 (1983).

32. Burchuladze, A. A., Pagava, S. V., Togonidze, G. I. \& Avtandilashvili, M. V. Radiocarbon and 11-Year Variations of Cosmic Rays. Radiocarbon 35, 347-350 (1993).

33. Weinstock, B. Carbon Monoxide: Residence Time in the Atmosphere. Science 166, 224-225 (1969).

34. Manning, M. R. et al. The Use of Radiocarbon Measurements in Atmospheric Studies. Radiocarbon 32, 37-58 (1990).

35. Damon, P. E., Kaimei, D., Kocharov, G. E., Mikheeva, I. B. \& Peristykh, A. N. Radiocarbon Production by the Gamma-Ray component of Supernova explosions. Radiocarbon 37, 599-604 (1995).

36. Dee, M., Pope, B., Miles, D., Manning, S. \& Miyake, F. Supernovae and Single-Year Anomalies in the Atmospheric Radiocarbon Record. Radiocarbon 59, 293-302 (2016).

37. Menjo, H. et al. Possibility of the detection of past supernova explosion by radiocarbon measurement. In 29th International Cosmic Ray Conference Pune 357-360 (2005).

38. Büntgen, U. et al. Tree rings reveal globally coherent signature of cosmogenic radiocarbon events in 774 and 993 CE. Nat. Commun. 9, 1-7 (2018).

39. Savard, M. M., Bégin, C., Marion, J., Arseneault, D. \& Bégin, Y. Evaluating the integrity of C and O isotopes in sub-fossil wood from boreal lakes. Palaeogeogr. Palaeoclimatol. Palaeoecol. 348-349, 21-31 (2012).

40. Kudsk, S. G. K. et al. What Is the Carbon Origin of Early-Wood? Radiocarbon 60, 1457-1464 (2018).

41. Dee, M. W. et al. Radiocarbon Dating at Groningen: New and Updated Chemical Pretreatment Procedures. Radiocarbon 00, 1-12 (2019).

42. Wijma, S., Aerts, A. T., van der Plicht, J. \& Zondervan, A. The Groningen AMS facility. Nucl. Instruments Methods Phys. Res. Sect. B Beam Interact. with Mater. Atoms 113, 465-469 (1996).

43. Aerts-Bijma, A. T., Meijer, H. A. J. \& Van Der Plicht, J. AMS sample handling in Groningen. Nucl. Instruments Methods Phys. Res. Sect. B Beam Interact. with Mater. Atoms 123, 221-225 (1997).

44. Savitzky, A. \& Golay, M. J. E. Smoothing and Differentiation of Data by Simplified Least Squares Procedures. Anal. Chem. 36, $1627-1639$ (1964).

\section{Acknowledgements}

This work was supported by a European Research Council grant (ECHOES, 714679). We would like to acknowledge the contribution to this research of the staff at the Centre for Isotope Research, Groningen, especially A. Aerts-Bijma, M. Bleeker, S. de Bruijn, F. Ghebru, S. Palstra, D. van Zonneveld, B. Verstappen-Dumoulin and P. Wietzes. This work was performed in part under contract with the Jet Propulsion Laboratory (JPL) funded by NASA through the Sagan Fellowship Program executed by the NASA Exoplanet Science Institute.

\section{Author contributions}

A.S. prepared the radiocarbon samples with the aid of M.K., performed the numerical analysis with the help of A.N., and wrote the paper. M.W.D. and B.J.S.P. conceived the idea and M.W.D. helped write the paper. A.M.S. and B.J.S.P. advised on theoretical aspects of cosmogenic isotope production. F.M. provided unpublished radiocarbon data from earlier work. E.J., P.D. and D.M. conducted the dendrochronological analyses and supplied the samples. All author were involved in editing the manuscript.

\section{Competing interests}

The authors declare no competing interests.

\section{Additional information}

Supplementary information is available for this paper at https://doi.org/10.1038/s41598-019-53296-X.

Correspondence and requests for materials should be addressed to A.S.

Reprints and permissions information is available at www.nature.com/reprints.

Publisher's note Springer Nature remains neutral with regard to jurisdictional claims in published maps and institutional affiliations. 
(c) (i) Open Access This article is licensed under a Creative Commons Attribution 4.0 International License, which permits use, sharing, adaptation, distribution and reproduction in any medium or format, as long as you give appropriate credit to the original author(s) and the source, provide a link to the Creative Commons license, and indicate if changes were made. The images or other third party material in this article are included in the article's Creative Commons license, unless indicated otherwise in a credit line to the material. If material is not included in the article's Creative Commons license and your intended use is not permitted by statutory regulation or exceeds the permitted use, you will need to obtain permission directly from the copyright holder. To view a copy of this license, visit http://creativecommons.org/licenses/by/4.0/.

(C) The Author(s) 2019 